\title{
Actividad antioxidante del gel a base de extracto de Origanum vulgare ¿importante para la salud bucal? Estudio preliminar
}

\author{
Antioxidant activity of Origanum vulgare extract gel. Is it important for oral health?. Preliminary study
}

Luis Galvez-Calla 1,a , María A. Alvarez-Páucar 2,b,c, Omar Alcázar-Aguilar ${ }^{3, b, d}$, Frank Mayta-Tovalino ${ }^{4, b, e}$, Felipe Lozano-Castro ${ }^{2, b, f}$, Edwin Cordova-Huayanay ${ }^{5, b, g}$, Roxana Revoredo-Morote ${ }^{2, b, h,}$

\section{RESUMEN}

Objetivo: Determinar la actividad antioxidante del extracto hidroalcohólico de Origanum vulgare a través de 03 métodos de ensayo DPPH, ABTS y FRAP. Material y métodos: La muestra recolectada de Origanum vulgare se secó, redujo el tamaño y se colocó a macerar 500 gramos de muestra seca en $1000 \mathrm{~mL}$ de etanol 97\% durante una semana. Pasado el tiempo se procedió a filtrar el macerado y se concentró en estufa. Se procedió a realizar las formulaciones. Estas diluciones, se sometieron a los análisis antioxidantes. Resultados: Método DPPH, el gel al $25 \%$ mostró un $\mathrm{IC}_{50}$ de $98,485 \mathrm{mg} / \mathrm{mL}$ equivalente a la dilución del 78,789\% y para el caso del estándar Trolox ${ }^{\circledR}$ presentó un $\mathrm{IC}_{50}$ de $2,48 \mu \mathrm{g} / \mathrm{mL}$. Método ABTS, la formulación de gel al $25 \%$ presentó un $\mathrm{IC}_{50}$ de 3,687 $\mathrm{mg} / \mathrm{mL}$ equivalente a una dilución de 77,75\% y para el estándar Trolox ${ }^{\circledR}$ presentó un $\mathrm{IC}_{50}$ de $2,99 \mu \mathrm{g} / \mathrm{mL}$, a diferencia de las otras formulaciones. Se evidenció relación entre el porcentaje de inhibición y concentración de las muestras con una correlación aceptada $\left(\mathrm{R}^{2}\right)$ para geles al 25\%, 50\%, 75\% de extracto de Origanum vulgare y Trolox ${ }^{\circledR}$ de 0,9972; 0,9987 y 0,9986 respectivamente. Método FRAP, observó acción rápida durante los 4 minutos, siendo

\footnotetext{
Departamento Académico de Ciencias Básicas, Facultad de Odontología, Universidad Nacional Mayor de San Marcos. Lima, Perú. Universidad Nacional Mayor de San Marcos. Lima, Perú.

Universidad Alas Peruanas. Lima, Perú.

Universidad Científica del Sur. Lima, Perú.

Departamento de Estomatología Rehabilitadora, Facultad de Odontología Universidad Nacional Mayor de San Marcos. Lima, Perú.

Doctor en Estomatología.

Docente

Doctor en Ciencias de la Salud; Especialista en Odontopediatría; Postgraduada en Competencias para la Formulación y Dirección de Proyectos de Investigación.

Magíster en Docencia Universitaria y Gestión Educativa; Especialista en Ortodoncia y Ortopedia Maxilar; Doctorando en Estomatología, Magíster en Estomatología; Especialista en Estadística en Investigación

Magíster en Estomatología; Especialista en Rehabilitación Oral; PostGraduado en Formulación y Dirección de Proyectos de Innovación Educativa.

g Maestro en Estomatología

h Especialista en Ortodoncia y Ortopedia Maxilar; Master en Gerencia Pública.
} 
$125 \mathrm{mg}$ de extracto contenido en el gel de $25 \%$ equivalente a $4 \mathrm{mg}$ de Trolox®. Conclusiones: Se determinó la actividad antioxidante equivalente al Trolox ${ }^{\circledR}$, mediante análisis antioxidante, con mejor poder de captación de radical libre promedio (Trolox/mg) de extracto y estuvo presente en el gel a base de Extracto de Origanum vulgare al $25 \%$.

PALABRAS CLAVES: antioxidantes, estrés oxidativo, Origanum vulgare, radicales libres, salud bucal.

\section{SUMMARY}

Objective: To determine the antioxidant activity of the hydroalcoholic extract of Origanum vulgare through DPPH, ABTS and FRAP essays. Material and Methods: The sample collected from Origanum vulgare was dried, reduced in size and placed to macerate 500 grams of dry sample in $1000 \mathrm{~mL}$ of $97 \%$ ethanol for one week. After time, the macerate was processed through a filter and concentrated in an oven. The formulations were carried out and the dilutions were analyzed with the antioxidant essays. Results: DPPH Method, the $25 \%$ gel showed an $\mathrm{IC}_{50}$ of $98.485 \mathrm{mg} / \mathrm{mL}$ equivalent to the dilution of $78.789 \%$ and for the Trolox ${ }^{\circledR}$ standard, it presented IC $_{50}$ of 2.48 $\mu \mathrm{g} / \mathrm{mL}$. ABTS Method, the $25 \%$ gel formulation presented $\mathrm{IC}_{50}$ of $3.687 \mathrm{mg} / \mathrm{mL}$ equivalent to a dilution of $77.75 \%$ and for the Trolox® standard it presented $\mathrm{IC}_{50}$ of $2.99 \mu \mathrm{g} / \mathrm{mL}$, a difference from the other formulations. The results evidenced a relation between the percentage of inhibition and concentration of the samples with an accepted correlation (R2) for the gels at $25 \%, 50 \%, 75 \%$ of extract of Origanum vulgare and Trolox® of 0.9972 ; 0.9987 and 0.9986 respectively. FRAP Method, with fast action during the 4 minutes, being $125 \mathrm{mg}$ of extract contained in the gel $25 \%$ equivalent to $4 \mathrm{mg}$ of Trolox®. Conclusions: It was determined that the antioxidant activity equivalent to Trolox ${ }^{\circledR}$, with antioxidant assays, with the best average free radical uptake power (Trolox $/ \mathrm{mg}$ ) of extract was present in the gel of Extract of Origanum vulgare 25\%.

KEY WORDS: antioxidants, free radicals, oral health, Origanum vulgare, oxidative stress.

\section{INTRODUCCIÓN}

Actualmente, diversos estudios demuestran la asociación entre Estrés Oxidativo y Enfermedades Orales, especialmente el papel de los radicales libres en el desarrollo de la enfermedad y la acción de los antioxidantes en su fase preventiva y curativa. Las células del sistema inmune crean radicales libres para matar bacterias y virus, pero si no hay un equilibrio (control ejercido por antioxidantes), estas células no se reconocen y llegan a dañar a las células sanas. Los radicales libres o metabolitos reactivos del oxígeno (MRO), son moléculas formados en el cuerpo durante el metabolismo celular normal o por la exposición a toxinas ambientales (contaminantes en el aire, alimentos y agua), estos radicales libres pueden ser dañinos, ya que poseen un electrón no apareado (configuración inestable), de extraordinaria reactividad y enorme capacidad para combinarse inespecíficamente con lípidos, proteínas, ácidos nucleicos y derivados de cada uno de ellos, importante en la patogenia de la enfermedad $(1,2)$.
Cuando la defensa antioxidante es insuficiente para proteger al organismo, ocurre ruptura del equilibrio entre sustancias antioxidantes y oxidantes a beneficio de los radicales libres, factor llamado estrés oxidativo, el exceso de oxígeno resulta potencialmente peligroso debido a la formación de MRO (1,3,4,5). El estrés oxidativo severo puede causar daño celular y muerte, implica presencia de patologías: aterosclerosis, psoriasis, diabetes mellitus, disfunción inmune, enfermedades orales, entre otros $(1,5,6)$. La oxidación celular tiene vinculación en el papel fisiológico y patogénico de las enfermedades orales, se ha demostrado la relación entre el estrés oxidativo y el proceso inflamatorio de enfermedades gingivales y periodontales, lesiones de mucosa oral, inflamación pulpar y trastornos temporomandibulares; en pacientes con enfermedad periodontal crónica, se observó efectos genotóxicos en la mucosa, aumento del estrés oxidativo y aumento en la producción de radicales libres (7). El envejecimiento y el desarrollo no son fases disímiles de la vida, el envejecimiento (fase final del desarrollo), si bien no es un fenómeno 
genéticamente programado, esto ocurre por el influjo del estrés oxidativo en el evento genético, aquíradica la importancia de la terapia antioxidante y de la dieta sobre las enfermedades (8). El consumo de nutrientes antioxidantes (compuestos no tóxicos: vitamina $\mathrm{E}$, betacaroteno, licopeno y selenio), reducen el riesgo de padecer cáncer y de las condiciones precancerosas, el consumo de frutas, verduras, la ingesta de polifenoles previene enfermedades no transmisibles $(9,10)$. Además, reduce la incidencia del cáncer oral (reduce el stress oxidativo) en personas que padecen enfermedades sistémicas.

Es importante, valorar la CA (capacidad de capturar un radical o reducir un agente antioxidante), donde se utilizan métodos basados en el monitoreo espectrofotométrico de la formación o desaparición de compuestos cromogénicos de naturaleza radical, entre ellos DPPH (2,2-difenil-1-picrylhydrazyl), ABTS 2,20-azino-bis (ácido 3-etilbenzo-tiazolina- 6sulfónico), ORAC (capacidad de absorción de radicales de oxígeno), FRAP (capacidad de reducción férrica de plasma), entre otros. Se sabe que, la determinación de polifenoles totales en una muestra, lo señala como un buen indicador de CA en alimentos (vegetales, frutas), plantas, estos métodos monitoreados por espectrofotometría UV-visible y algunos de ellos dan una medición directa de CA (métodos ABTS y DPPH), porque se basan en una captura directa del radical por la muestra; otros ensayos (métodos FRAP y Folin-Ciocalteu) dan una medición indirecta de CA (10), incluso estas pruebas (métodos Folin-Ciocalteu, DPPH y FRAP), sirven para indicar la cantidad necesaria de antioxidante natural que se debe agregar a productos cárnicos (11). Una posible solución para el cuidado de la salud oral sería, el uso de antioxidantes de determinadas plantas, que ayuden a los antioxidantes endógenos a neutralizar el estrés oxidativo hasta la aplicación de productos naturales. El objetivo de nuestro estudio preliminar, fue determinar la capacidad antioxidante de los geles a base de extracto hidroalcohólico de Origanum vulgare.

\section{MATERIAL Y MÉTODOS}

Se efectuó un estudio in vitro y de tipo transversal, sobre la actividad antioxidante del gel formulado con el extracto de la planta entera Origanum vulgare
(OV) a concentraciones de 25\%, 50\%, 75\% y $100 \%$, empleando pruebas de captura de radicales libres en métodos DPPH, ABTS y FRAP.

\section{Recolección de la planta}

Las hojas del Origanum vulgare fueron recogidas de parcelas artesanales ubicadas en el distrito de Ilaballa, provincia de Jorge Basadre, departamento de Tacna.

\section{Herbal classification}

El análisis de la planta fue realizado en el Laboratorio de Botánica y Recursos Genéticos, Facultad de Ciencias Agropecuarias, Universidad Nacional Jorge Basadre Grohmann (Tacna). La sistemática de las especies según Sistema de Clasificación de APG III (Angiosperm Phylogen Group III, 2009) es como sigue: Orden: Lámidas, Familia: Lamiaceae, Género: Origanum, Especie: vulgare. Nombre científico: Origanum vulgare.

\section{Preparación del extracto antioxidante natural.}

Se recolectaron $2 \mathrm{~kg}$ de hojas de Origanum vulgare y transportadas al Laboratorio de Farmacognosia, Facultad de Farmacia y Bioquímica de la Universidad Nacional Mayor de San Marcos (Lima), previamente seleccionadas, sólo hojas en buenas condiciones, sin daño, ni atacadas por hongos, ni decoloradas, ni marchitadas. Se lavó las hojas con agua destilada y se desinfectó con hipoclorito de sodio 0,5\%. Las hojas fueron colocadas en papel Kraft, luego se llevó a secar a la estufa de circulación de aire por convección forzada a $40^{\circ} \mathrm{C}$ por 48 horas. Enseguida del secado, las hojas se pulverizaron mediante un mortero. El polvo fue almacenado en un frasco de vidrio ámbar de boca ancha y pesó con exactitud 500 gr de Origanum vulgare. Luego se maceraron en un frasco de vidrio ámbar de boca ancha de $1 \mathrm{~L}$ de capacidad y se añadió etanol $96^{\circ}$ cantidad suficiente hasta cubrir la muestra por sobre $2 \mathrm{~cm}$ de altura. Se mezcló bien, se tapó el recipiente y se maceró por 7 días, agitándose 15 minutos, dos veces al día. Luego el macerado fue concentrado en una estufa hasta sequedad. Posteriormente, se procedió a realizar las formulaciones y realizar los análisis antioxidantes DPPH, ABTS y FRAP. 
Determinación de la capacidad antioxidante in vitro del extracto de la planta entera

\section{a. Método de DPPH (actividad secuestradora del radical 2,2-difenil-1-picrilhidrazilo)}

Se realizó en las instalaciones de la Facultad de Farmacia y Bioquímica, Universidad Nacional Mayor de San Marcos, Servicio Académico Asistencial de Análisis Clínico. El DPPH, es un indicador para medir la capacidad de secuestro de cualquier compuesto con actividad antioxidante. La reacción desarrolla un cambio de color violeta a amarillo a medida que disminuye la absorbancia a $515 \mathrm{~nm}$. Se siguió la metodología descrita por Portes et. al., (12), siendo ampliamete usado en difrentes muestras y de fácil preparación.

Se preparó el Reactivo DPPH de la siguiente manera: Se pesó 39,43mg aproximadamente en la balanza analítica y se disolvió con metanol hasta $100 \mathrm{~mL}$ para obtener una concentración de $1 \mathrm{mM}$. Se usó como blanco metanol. Para acceder al reactivo de trabajo, se tomaron $500 \mu \mathrm{L}$ de muestra con $500 \mu \mathrm{L}$ de reactivo DPPH y se hizo reaccionar por 30 minutos a temperatura ambiente en oscuridad. Pasado el tiempo se leyó a 540nm.

b. Método de ABTS (ácido 2,2- azinobis-3-etil benzotiazolina-6-sulfónico)

Se realizó en las instalaciones de la Facultad de Farmacia y Bioquímica, Universidad Nacional Mayor de San Marcos, Servicio Académico Asistencial de Análisis Clínico. Se siguió la metodología según Kuskoski et. al., (13), debido a su simplicidad al momento de la preparación del radical libre y a su amplia aplicabilidad. Método monitoreado por espectrofotometría UV-visible y algunos de ellos dan una medición directa sobre la capacidad de capturar un radical o reducir un agente antioxidante (junto al método ABTS está la prueba DPPH).

Se preparó la solución A: Se disolvió 38.4 mg de ABTS en $10 \mathrm{~mL}$ de agua destilada y la solución B: Se disolvió 378,4 mg de persulfato de potasio en $10 \mathrm{~mL}$ de agua destilada. Para acceder al reactivo de trabajo, en un beacker limpio se tomó $10 \mathrm{~mL}$ de sol. A y se mezcló con $176 \mu \mathrm{L}$ de sol. B. Se dejó reaccionar por 16 horas en total oscuridad, concluido el tiempo se diluyó el reactivo de trabajo con agua destilada hasta obtener una absorbancia de $0,7 \pm 0,05$ a $734 \mathrm{~nm}$ y se usó como blanco agua destilada. Para la reacción, se tomó en un beacker limpio $20 \mu \mathrm{L}$ de muestra y se hizo reaccionar con $980 \mu \mathrm{L}$ de reactivo ABTS por 10 minutos en oscuridad a temperatura ambiente y se usó como blanco agua destilada. Concluido el tiempo se leyó a 734nm. Se usó como unidad de medida estándar el Trolox. Los resultados fueron expresados en porcentaje de inhibición de la formación de radicales ABTS.

c. Método FRAP (poder antioxidante de la reducción férrica, por sus siglas en inglés)

Se realizó en las instalaciones de la Facultad de Farmacia y Bioquímica, Universidad Nacional Mayor de San Marcos, Servicio Académico Asistencial de Análisis Clínico. Se siguió la metodología según Sonawane et. al., (14), debido a que es el más utilizado para este tipo de muestras semisólidas miscibles con agua. Método monitoreado por espectrofotometría UV-visible y medición indirecta sobre la capacidad de capturar un radical o reducir un agente antioxidante, su fundamento radica en la capacidad para reducir el complejo de fierro.

Se preparó la solución A: Buffer acetato $0,3 \mathrm{M}$ a un pH 3,8 (4,9 gramos de acetato de sodio $+24 \mathrm{~mL}$ ácido acético glacial, completar con agua destilada hasta $1 \mathrm{~L})$, luego la solución B: 0,01M TPTZ $(312,33 \mathrm{mg}$ TPTZ) en $0,04 \mathrm{M} \mathrm{HCl}(320 \mu \mathrm{L} \mathrm{HCl} 37 \%$ hasta $100 \mathrm{~mL}$ agua) y por último la solución $\mathrm{C}: 0,02 \mathrm{M} \mathrm{FeCl}_{3} \cdot 6 \mathrm{H}_{2} \mathrm{O}$ (540,6 $\mathrm{mg} \mathrm{FeCl}_{3} \cdot 6 \mathrm{H}_{2} \mathrm{O}$ hasta $100 \mathrm{~mL}$ agua). Para acceder al reactivo de trabajo, en un frasco ámbar de tapa rosca con capacidad suficiente se mezcló $1 \mathrm{~L}$ de sol. A $+100 \mathrm{~mL}$ de sol. $\mathrm{B}+100 \mathrm{~mL}$ de sol. $\mathrm{C}$, luego se dejó reaccionar por 1 hora a $37^{\circ} \mathrm{C}, 4$ horas a temperatura ambiente. Para la reacción, se tomó 800 $\mu \mathrm{L}$ de reactivo de trabajo y se hizo reaccionar con $200 \mu \mathrm{L}$ de muestra, por 4 minutos de manera cinética con intervalos de 1 minuto, la absorbancia durante el ensayo fue de 593nm. Se usó como blanco agua destilada y diluyente de muestra en las proporciones de $4: 1$.

\section{RESULTADOS}

La actividad antioxidante del gel a base de extracto de orégano fue evaluada por los métodos de 
DPPH y del radical catiónico ABTS; los resultados de la muestra fueron comparados frente al Trolox ${ }^{\circledR}$, un análogo soluble a la vitamina $\mathrm{E}$, con características antioxidantes. Los ensayos evaluados en las formulaciones del gel al 25; 50; $75 \%$ se realizaron por quintuplicado, y por triplicado para el estándar Trolox®. Se calculó el $\mathrm{IC}_{50}$ (concentración inhibitoria media) en $\mu \mathrm{g}$ de extracto contenido en la formulación $/ \mathrm{mL}$, este dato corresponde a la cantidad de extracto en la dilución del gel que reduce en un 50\% la absorbancia de la solución contenedora de radicales. Para el DPPH, la primera formulación presentó un
$\mathrm{IC}_{50}$ de $98,485 \mathrm{mg} / \mathrm{mL}$ equivalente a la dilución de $78,789 \%$ del gel al 25\%. Para las formulaciones del 50 y $75 \%$ presentaron $\mathrm{IC}_{50}$ de 131,742 y $119,037 \mathrm{mg} /$ $\mathrm{mL}$ respectivamente, equivalentes a las diluciones del $52,69 \%$ y $31,734 \%$ de sus respectivas formulaciones. Para el caso del estándar Trolox ${ }^{\circledR}$, presentó un $\mathrm{IC}_{50}$ de 2,48 ug/mL. La asociación entre los elementos evaluados como Porcentaje de inhibición y concentración de las muestras obtuvo una correlación aceptada, con Coeficientes de Pearson $\left(\mathrm{R}^{2}\right)$ para el gel al $25 ; 50 ; 75 \%$ y Trolox ${ }^{\circledR}$ de: 0,$9941 ; 0,9988 ; 0,9974$ y 0,9991 respectivamente (gráfico 1 y gráfico 2).

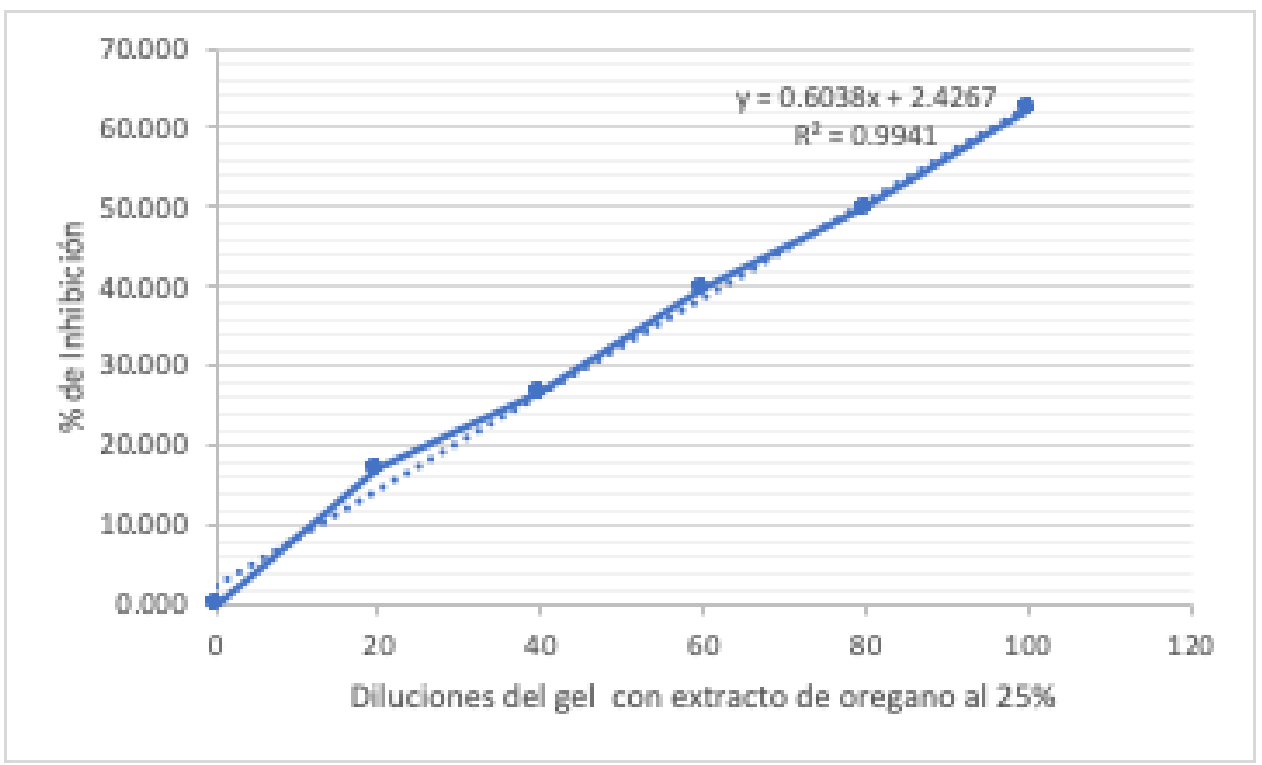

Gráfico 1. Porcentaje de Inhibición del radical DPPH vs Diluciones del gel al 25\%

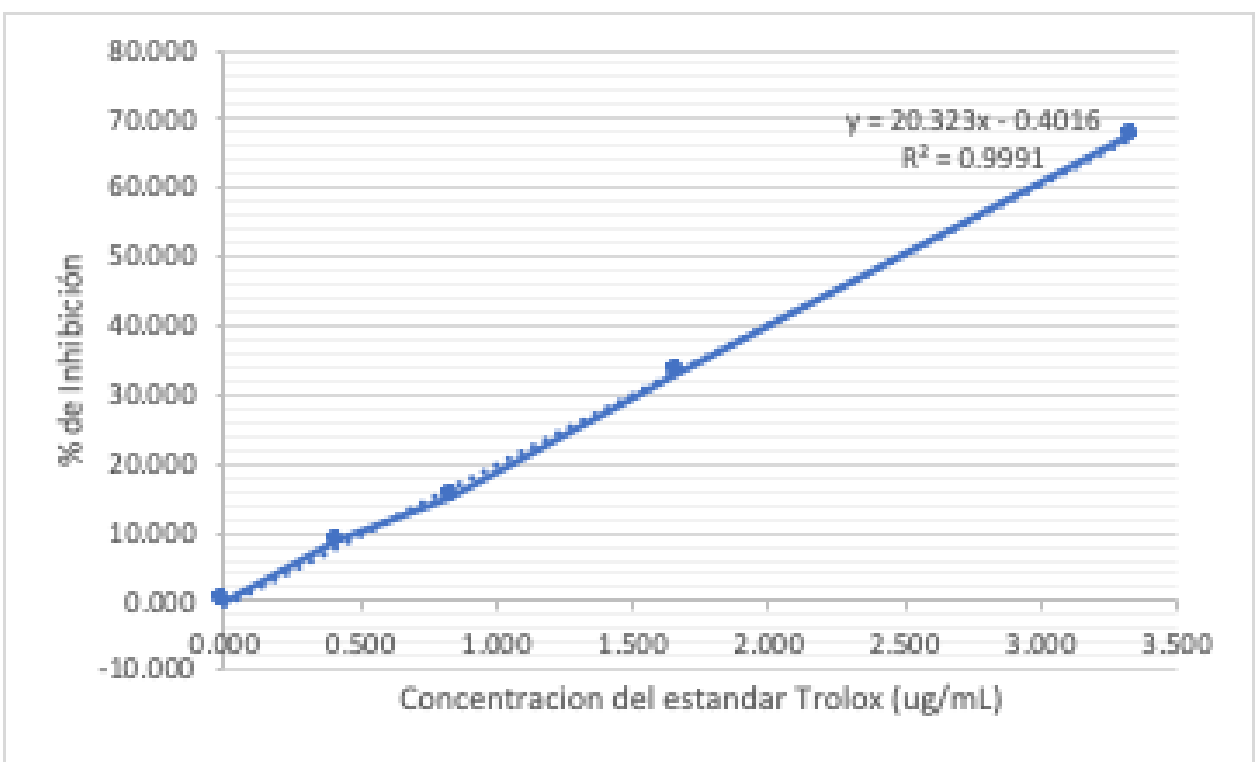

Gráfico 2. Porcentaje de Inhibición del radical DPPH vs concentración de Trolox® 
Para las formulaciones del 25; 50 y $75 \%$ mediante el ensayo del radical catiónico de ABTS presentaron $\mathrm{IC}_{50}$ de 3,$687 ; 5,329$ y $5,561 \mathrm{mg} / \mathrm{mL}$ equivalentes a las diluciones de 77,75; 44,951 y 37,068 respectivamente. Para el estándar Trolox ${ }^{\circledR}$ el $\mathrm{IC}_{50}$ fue de $2,99 \mu \mathrm{g} / \mathrm{mL}$. La asociación entre el porcentaje de inhibición y concentración de las muestras presenta una correlación aceptada, con Coeficientes de Pearson $\left(\mathrm{R}^{2}\right)$ para el gel al $25 ; 50 ; 75 \%$ y Trolox ${ }^{\circledR}$ de: 0,9972 ; 0,9987; 0,9986 y 0,9986 respectivamente (gráfico 3 y gráfico 4). Por otro lado, la diferencia de medias de la absorbancia es significativa en el nivel 0,05 (ANOVA Post hoc Tukey) del Trolox frente a las pruebas ABTS y DPPH.

Los TEAC (Capacidad antioxidante equivalente al Trolox), mediante el método del ABTS, fue de 0,8125 $\mu \mathrm{g}$ de Trolox/mg de extracto presentes en gel al $25 \%$. Y el TEAC según método de DPPH es de $0,025 \mu \mathrm{g}$ de Trolox/mg de extracto presente en el gel al $25 \%$.

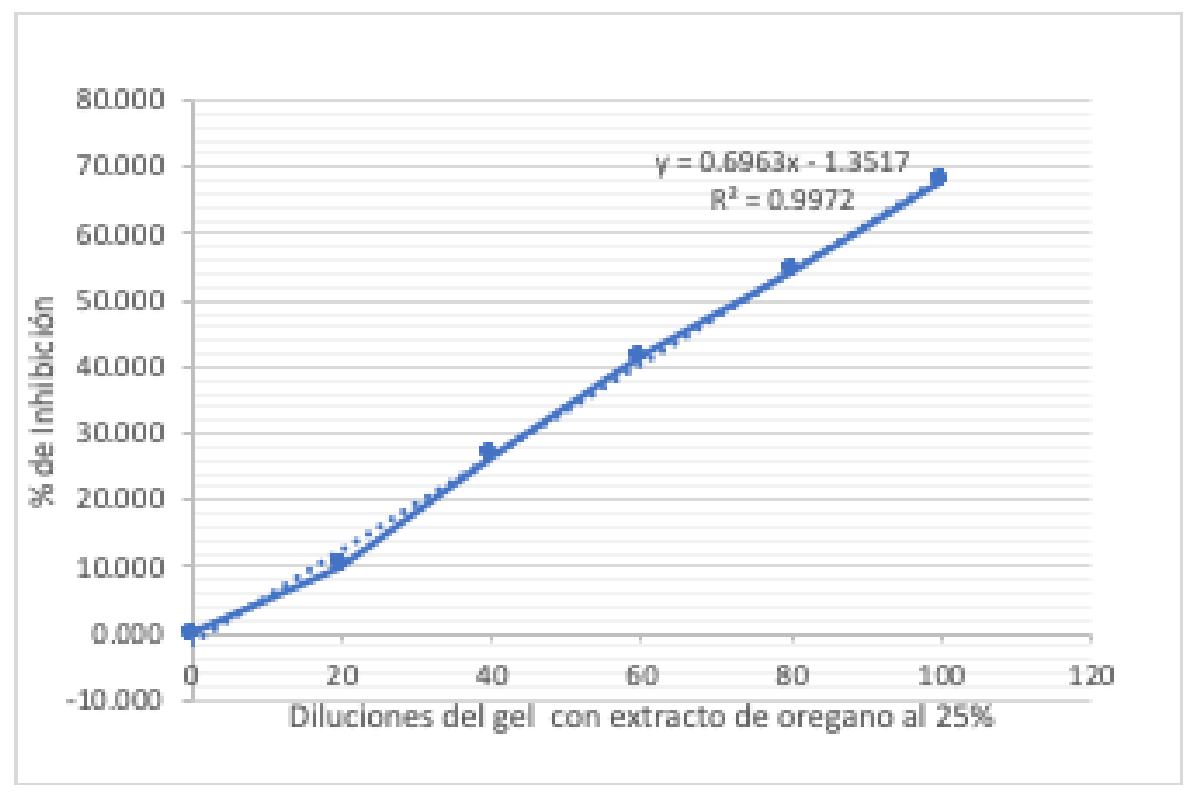

Gráfico 3. Porcentaje de Inhibición del radical ABTS vs Diluciones del gel al 25\%

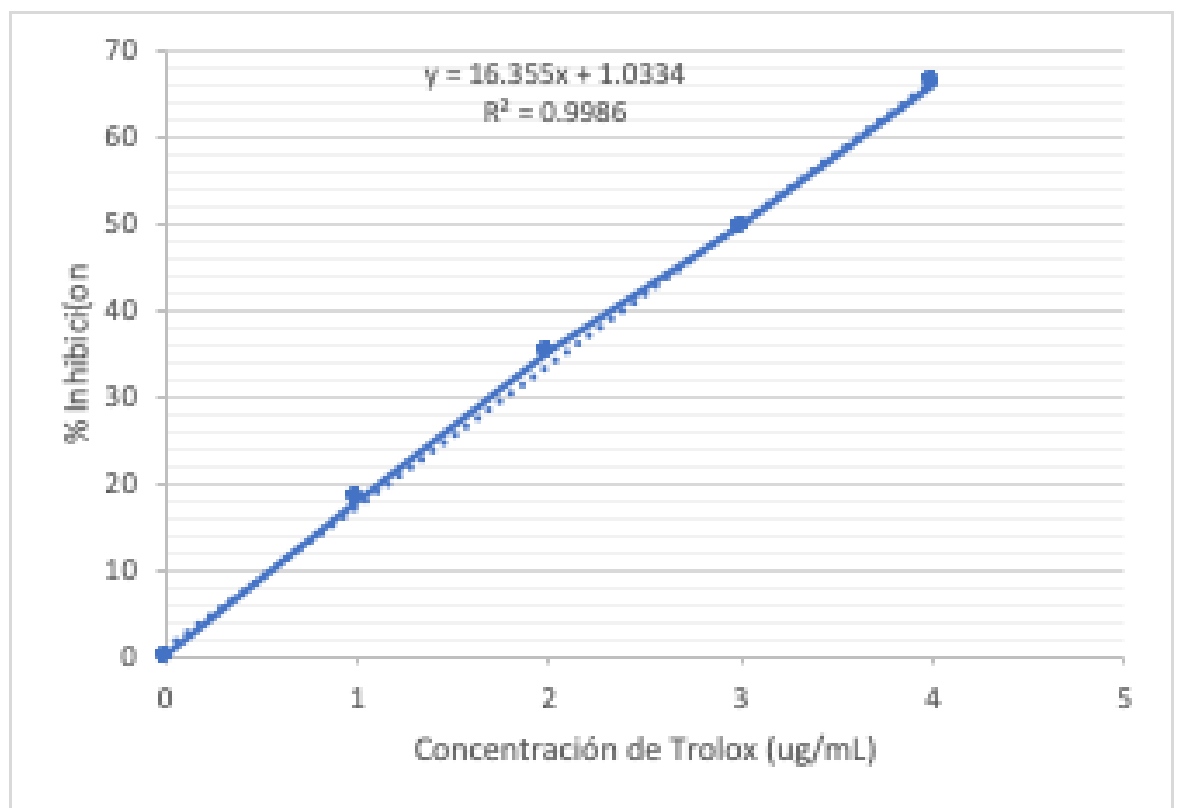

Gráfico 4. Porcentaje de Inhibición del radical ABTS vs concentraciones de Trolox 


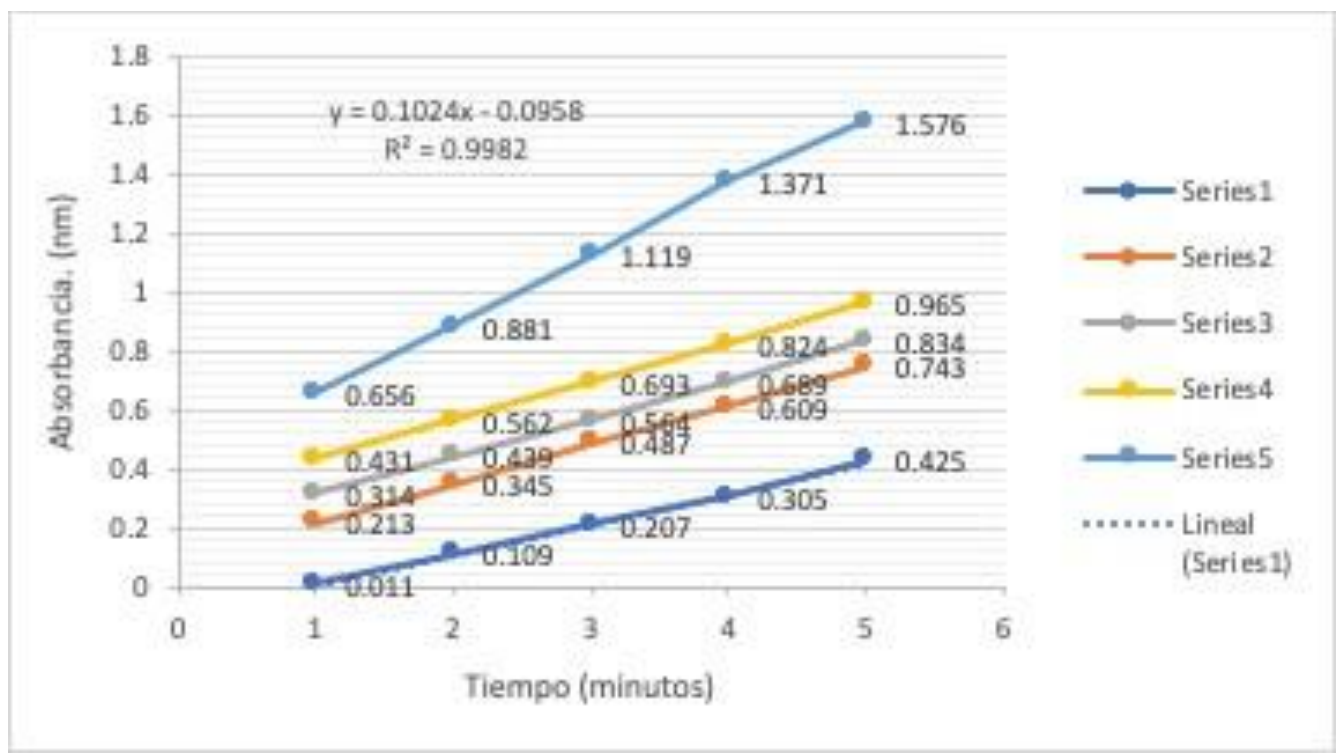

Gráfico 5. Ecuación de la recta de la curva de calibración de Trolox.

En Método FRAP, el análisis se realizó de manera cinética durante 5 minutos, el poder reductor de hierro de la muestra fue excelente, muy potente al inicio, pero se detuvo, para mantenerse en el tiempo (acción rápida), logrando superar al Trolox ${ }^{\circledR}$ los primeros 4 minutos y luego su actividad se mantuvo de manera indefinida. Observándose esa tendencia en todas las series 1-5, equivalentes a 2, 4, 6, 8 y 10mg/ $\mathrm{mL}$ respectivamente. En este análisis no se aplicó ninguna fórmula para calcular el poder reductor; sino que se realizó mediante interpolación usando la curva de calibración de Trolox ${ }^{\circledR}$ (gráfico 5). Por lo tanto, $125 \mathrm{mg}$ de extracto contenido en el gel al $25 \%$ equivale a $4 \mathrm{mg}$ de Trolox ${ }^{\circledR}$.

\section{DISCUSIÓN}

La Organización Mundial de la Salud (OMS) en el 2020, cita que la tasa de morbilidad por enfermedades bucodentales y otras enfermedades no transmisibles pueden reducirse por intervenciones de salud pública dirigidas a los factores de riesgo más comunes, mediante una reforma en los sistemas de salud, implica prestar atención a la prevención y tratamientos menores, descartando tratamientos dentales invasivos; la mayoría de enfermedades y trastornos bucodentales conllevan factores de riesgo modificables (consumo de: alcohol, tabaco y alimentación malsana ricos en azúcares libres) comunes a las 04 principales enfermedades no transmisibles como: enfermedades cardiovasculares, cáncer, enfermedades respiratorias y diabetes mellitus (15). Recientes estudios demuestran que la oxidación celular tiene vinculación en el papel fisiológico y patogénico de las enfermedades orales, por ende, aumenta el riesgo de enfermedades no transmisibles. En Perú, la salud bucal constituye problema de Salud Pública.

La comunidad científica muestra alto interés en los compuestos bioactivos y su relación en la disminución del riesgo de enfermedades no transmisibles. La aplicación de los métodos de detección in vitro, como: DPPH, ABTS podrían ayudar a diseñar futuros estudios de investigación sobre "compuestos bioactivos" (16). El Origanum vulgare contiene fuentes ricas en compuestos bioactivos, incluidos los ácidos fenólicos y los flavonoides como los más abundantes (17). La medición de concentraciones de fenoles totales, flavonoides y otros quimiotipos como carvacrol y timol, se realizan mediante espectrofotometría UV y ofrece un índice químico rápido, sin embrago, las técnicas cromatográficas sirven para establecer la actividad de la estructura (11). La evaluación de la actividad antioxidante se determina mediante la comparación de los diferentes ensayos empleados $(18,19)$.

El presente estudio usó métodos: ABTS, DPPH y FRAP para determinar la magnitud de los componentes fenólicos que contiene el Origanum vulgare (OV), para captar los radicales libres generados contra el proceso 
de oxidación. Los estudios indican que los métodos ABTS y DPPH, presentan alta estabilidad en ciertas condiciones y miden 02 mecanismos: transferencia de electrón y transferencia de átomos de hidrógeno $(18,20,21)$, aunque también revelan diferencias, el ABTS tiene la ventaja que su espectro indica máximos de absorbancia a 414, 654, 754 y $815 \mathrm{~nm}$ en medio alcohólico, mientras que el DPPH observa un pico de observancia a $515 \mathrm{~nm}$ (13). Mientras que, el FRAP mide la capacidad reductora del hierro en las condiciones de extracción de la muestra (22), y evalúa la cuantificación de transferencia de electrones $(18,20,21)$. Aunque, algunos estudios revelaron que la prueba Folin-Ciocalteu y $\mathrm{AlCl} 3$ se utilizan para evaluar los fenólicos y flavonoides totales (16), otros estudios registraron que, la actividad antioxidante in vitro y el contenido fenólico total medidos con el reactivo Folin-Ciocalteu no son los apropiados (23). La cromatografía líquida-espectroscopía de masas (LCMS) o el método HPLC, aún no son métodos estándar comunes, para identificar valores antioxidantes en la investigación científica, estas técnicas de vanguardia, identifican y cuantifican con precisión el contenido de compuestos bioactivos, especialmente fenólicos en alimentos y extractos herbales; sin embargo, los métodos de detección espectrofotométrica se usan para caracterizar materiales y dar una idea del contenido fenólico total (24). El presente trabajo, optó por el ensayo in vitro, porque los ensayos in vivo presentan inconvenientes y adaptabilidad en la respuesta al aumento del stress oxidativo $(10,13)$.

El presente estudio, optó por evaluar la actividad antioxidante (captación del radical libre) del extracto hidroalcohólico de $\mathrm{OV}$, considerando que, la composición agua/etanol, se encuentra en el extracto hidroalcohólico (HA) en sus diferentes concentraciones. La concentración DPPH en el medio de reacción se calculó a partir de la curva de calibración, determinada por regresión lineal $(\mathrm{r}$ $=0.994): Y=0,6038 X-2,4267$, el porcentaje de DPPH restante contra la concentración del extracto se trazó para obtener la cantidad de antioxidante necesaria para disminuir la concentración inicial de $\mathrm{DPPH}$ en un $\mathrm{IC}_{50}$. Coincidiendo con Cavero et. al., en el 2006 (25), que también siguió su curva de calibración, siendo su determinación por regresión lineal $(r=0.999)$ : $Y=0,0247 X-0,0029$; asimismo, observó, cuánto menor es el $\mathrm{IC}_{50}$, mayor es el poder antioxidante y cada determinación se repitió 2 veces, a diferencia de nuestro trabajo, que los ensayos evaluados en las formulaciones del gel 25; 50; $75 \%$ se corrió 5 veces, y para el estándar Trolox ${ }^{\circledR} 3$ veces, su estudio reportó valores del $\mathrm{IC}_{50}$ entre 53,8 y 118,3 $\mu \mathrm{g} / \mathrm{mL}$ en la fracción 1 y entre 69,4 y $169,7 \mu \mathrm{g} / \mathrm{mL}$ en la fracción 2. Sin embargo, Moghrovyan et. al., en el 2019 (26), registró valores más bajos en el DPPH, con un $\mathrm{IC}_{50}$ de $19.97 \mu \mathrm{g} / \mathrm{mL}$ en el extracto etanólico de OV, infiriendo mayor poder antioxidante respecto al anterior trabajo, siguiendo esa misma tendencia, nuestro trabajo refirió un $\mathrm{IC}_{50}$ de $2,48 \mu \mathrm{g} / \mathrm{mL}$ para el caso estándard Trolox ${ }^{\circledR}$ en el gel de extracto HA de orégano al $25 \%$ (muestra que obtuvo mejores resultados) y una capacidad antioxidante equivalente al Trolox de $0,025 \mu \mathrm{g}$ TE/mg de extracto HA presente en el gel al 25\%. Fierescau et. al., en el 2018 (27), mostró importante actividad antioxidante de hierbas silvestres como: Momordica charantia L., Humulus lupulus L., Origanum vulgare L. entre otros, con un DPPH para OV de $173.67 \pm 6.91 \mu \mathrm{g} \mathrm{TE} / \mathrm{mg}$, valor alto respecto a nuestro estudio, que podría deberse al empleo del extracto de metanol de OV. Por otro lado, Texeira et. al., en el 2013 (28), mostró pobre actividad antioxidante del aceite esencial de $\mathrm{OV}$, del extracto de agua fría con moderada actividad, el extracto etanólico presentó un fuerte valor y el extracto de aguacaliente observó la mayor actividad, de acuerdo al índice AAI (Indice de Actividad Antioxidante). Existen resultados controversiales respecto a 02 variedades de Orégano, Méabed et. al., en el 2018 (29), observó que el contenido total fenólicos, flavonoides y actividad antioxidante de DPPH del extracto de Origanum majorana, fue de $12,34 \pm 0,1 \mathrm{mg} / \mathrm{mL}$, valor alto que se podría inferir en menor activdad antioxidante, aunque los resultados pueden ser controversiales, ha sido ampliamente corroborada la acción antioxidante del OV.

Respecto al ensayo ABTS, el presente trabajo observó que el $\mathrm{IC}_{50}$ para el gel elaborado al $25 \%$ con el extracto de orégano, requirió la dilución al 73,74\% equivalente a $3,68 \mathrm{mg} / \mathrm{mL}$ de extracto $\mathrm{HA}$ de OV; para el caso estándard Trolox ${ }^{\circledR}$ presentó un $\mathrm{IC}_{50}$ de 2,99 $\mu \mathrm{g} / \mathrm{mL}$ y una capacidad antioxidante equivalente al Trolox de $0,8125 \mu \mathrm{g}$ TE/mg de extracto presentes en el gel al 25\%. Fierescau et. al., en el 2018 (27) hallaron un alto valor en la prueba ABTS de 422,06 $\pm 9,61 \mu \mathrm{g}$ $\mathrm{TE} / \mathrm{mg}$, este resultado podría deberse al empleo del extracto de OV obtenido del metanol, sin embargo, el estudio de Chun et. al., en el 2005 (30), notó que 
el porcentaje de inhibición de ABTS en muestras de orégano, fue mayor en extractos de agua que en extractos de etanol, lo que indica, la sensibilidad del ensayo hacia los antioxidantes solubles en agua.

Con el método FRAP, el poder reductor de hierro de la muestra fue excelente, potente y de acción rápida, los primeros 4 minutos, luego su actividad se detuvo de manera indefinida, esto indica que se logró superar el Trolox®. Se halló $125 \mathrm{mg}$ de extracto HA de Origanum vulgare contenido en el gel de $25 \%$ y equivale a $4 \mathrm{mg}$ de Trolox. El estudio de Texeira et. al., en el 2013 (28), reveló una mayor actividad antioxidante en el extracto de aceite esencial y en el extracto de agua fría, seguido del extracto de agua caliente y por último en el extracto etanólico de OV, sin embargo, el análisis de poder reductor mostró estadísticamente una mayor actividad antioxidante en el extracto de agua caliente, seguido del extracto etanólico, el extracto de agua fría y el aceite esencial, siendo el valor referencial FRAP, para el extracto etanólico de 12,6 $\pm 0,1 \mu \mathrm{M} \mathrm{Fe} \mathrm{F}^{+2} / \mathrm{g}$, sin embargo, Fierescau et. al., en el 2018 (27), registró 9,64 \pm 0,96 $\mathrm{mM} \mathrm{Fe} / 2 / \mathrm{mg}$, este alto valor, podría deberse al empleo del extracto de OV obtenido del metanol.

Aunque, la capacidad antioxidante de una muestra compuesta de varias moléculas electroactivas, no lo proporciona la suma de las capacidades antioxidantes individuales de sus componentes, sino del tipo de suelo (microambiente) que la nutre, periodo de floración, periodo de cosecha, entre otros factores, obteniéndose un efecto sinérgico o inhibitorio. La recolección de la muestra del presente trabajo, se realizó en el periodo de floración (mes de julio).

A nivel oral, el aumento del estrés oxidativo puede provocar dentinogénesis, mediante la disminución de la actividad de las células de la pulpa dental; pero la davallilactona (antioxidante derivado de hongos de origen natural) reduce la producción de peróxido de hidrógeno y otras moléculas inflamatorias, formación de MRO y alteraciones en la mineralización de la dentina. En periodontitis (infección bacteriana que produce inflamación crónica de la encía), se observa aumento de: flujo de MRO, de respuesta antibacteriana de neutrófilos, macrófagos, asimismo, aumento de concentración del producto peroxidación lipídica 8-isoprostano en pacientes con periodontitis crónica; las concentraciones séricas de MRO se asociaron con títulos de anticuerpos altos para bacterias periodontales: $P$. gingivalis, $P$. intermedia $y$ E. corrodens y en la enfermedad de Behçet (proceso inflamatorio crónico, con úlceras aftosas orales, úlceras genitales y uveítis recurrente) se observa trastornos en leucocitos activados y producción alta de MRO (4).

Recientemente, el estudio de los antioxidantes (ascorbato de sodio al 10\%, extracto de semilla de uva al $10 \%$, alfa tocoferol, extracto de semilla de pino al $10 \%$, gel de aloe vera, entre otros) abarca terapia restaurativa, periodontal, ortodóntica y prótesis dental, siendo capaz de modificar el stress oxidativo (1), en tratamiento estético, como maniobra previa de adhesión al esmalte tras el blanqueamiento dental, donde antioxidantes (ascorbato de sodio o alfa tocoferol) podrían reducir la presencia de oxígeno luego del blanqueamiento, recuperando valores de adhesión originales, siendo controversial para otros autores (31).

La aplicación biomédica de antioxidantes naturales está limitada por su escasa biodisponibilidad y estabilidad para cruzar la barrera transdérmica, se han ideado sistemas de administración y liberación controlada. Los productos fitoterapéutico en gel, pueden constituir una alternativa de tratamiento, que brindaría protección y beneficios sobre los factores de riesgo comunes a las enfermedades no transmisibles y enfermedades orales, como en el caso de las lesiones de la mucosa oral.

El presente trabajo observó actividad antioxidante del gel a base de extracto hidroalcohólico de Origanum vulgare, mediante las pruebas DPPH, ABTS FRAP, donde el mejor poder de captación de radical libre promedio (Trolox/mg) lo obtuvo el gel al $25 \%$.

\section{Correspondencia:}

Luis Hernando Galvez Calla.

Correo electrónico: lgalvezc@unmsm.edu.pe

\section{REFERENCIAS BIBLIOGRÁFICAS}

1. Valdés JM, Padrón R, El Ghannam Y. Estrés oxidativo en las enfermedades bucales. Rev Cubana Estomatol. 2016; 53 (4): 1-8.

2. Bhateja S. Role of antioxidants in oral medicine. Int J Pharm Sci Res. 2012; 3 (7): 1971-1975. 
3. Singh N, Niyogi R, Mishra D, Sharma M, Singh D. Antioxidants in oral health and diseases: Future Prospects. JDMS. 2013; 10 (3): 36-40.

4. Kesarwala AH, Krishna MC, Mitchell JB. Oxidative stress in oral diseases. Oral Dis. 2016; 22 (1):9-18.

5. Pedraza J, Eugenio D, Molina E. Uso de Activadores Naturales de Nrf2 en diversas condiciones patológicas. En: Cárdenas C, González M, Guevara A, Lara R, Matuz D, Molina E, Torres PV. Mensaje Bioquímico. México DF: Facultad de Medicina, Universidad Nacional Autónoma de México; 2016.p. 211-230.

6. Guija-Poma E, Inocente-Camones MA, PoncePardo J, Zarzosa-Norabuena E. Evaluación de la técnica 2,2-Difenil-1-Picrilhidrazilo (DPPH) para determinar capacidad antioxidante. Horiz Med. 2015; 15(1): 5760.

7. Gómez J, Morales G, Ortiz Y, et al. Disminución del daño nuclear y oxidativo al ADN en pacientes con periodontitis después de la ingesta de ácido fólico. Rev Mex Periodontol. 2015; 6(3):121-128.

8. Rodríguez T, Peña M, Gómez N, Santisteban L, Hernández M. Estrés oxidativo: genética, dieta y desarrollo de enfermedades. Correo Científico Médico de Holguín. 2015; 19(4): 690-705.

9. Oroian M, Escriche I. Antioxidants: Characterization, natural sources, extraction and analysis. Food Res Int. 2015; 74: 10-36.

10. Hoyos-Arbeláez J, Vázquez M, Contreras-Calderón J. Electrochemical methods as a tool for determining the antioxidant capacity of food and beverages: A review. Food Chemistry. 2017; 221: 1371-1381.

11. Fernandes RPP, Trindade MA, Tonin FG, et al. Evaluation of oxidative stability of lamb burger with Origanum vulgare extract. Food Chem. 2017; 233: 101-109.

12. Portes W, Gardrat C, Castellan A. A comparative study on the antioxidant properties of tetrahydrocurcuminoids and curcuminoids. Tetrahedron 2007; 63 (37): 90929099.

13. Kuskoski EM, Asuero AG, Troncoso AM, ManciniFilho J, Fett R. Aplicación de diversos métodos químicos para determinar actividad antioxidante en pulpa de frutos. Cienc. Tecnol Aliment. 2005; 25(4): 726-732.

14. Sonawane SK, Arya SS. Citrullus lanatus protein hydrolysate optimization for antioxidant potential. Food Measure. 2017; 11: 1834-1843.

15. Organización Mundial de la Salud. Salud bucodental. Ginebra: Organización Mundial de la Salud; 2020. (Fecha de acceso: 25 de marzo 2020). Disponible en: https://www.who.int/es/news-room/fact-sheets/detail/ oral-health

16. Granato D, Shahidi F, Wrolstad R. et al. Antioxidant activity, total phenolics and flavonoids contents:
Should we ban in vitro screening methods? Food Chem. 2018; 264: 471-475.

17. Gayoso L, Roxo M, Cavero RY, et al. Bioaccessibility and biological activity of Melissa officinalis, Lavandula latifolia and Origanum vulgare extracts: Influence of an in vitro gastrointestinal digestion. Journal of Functional Foods. 2018; 44: 146-154.

18. García-Pérez JS, Cuéllar-Bermúdez SP, de la CruzQuiroz R. et al. Supercritical CO2 based tailor made valorization of Origanum vulgare L extracts: A green approach to extract high-value compounds with applied perspectives. J Environ Manag. 2019; 232: 796-802.

19. Karadag A, Ozcelik B, Saner S. Review of Methods to Determine Antioxidant Capacity. Food Anal. Methods. 2009; 2(1):41-60.

20. Kedare SB, Singh RP. Genesis and development of DPPH method of antioxidant assay. J Food Sci Technol. 2011; 48(4):412-22.

21. Krishnaiah D, Sarbatly R, Nithyanandam R. A review of the antioxidant potential of medicinal plant species. Food and Bioproducts Processing. 2011; 89(3): 217233.

22. Carbonel KN, Suárez SA, Salas AI. Características fisicoquímicas y capacidad antioxidante in vitro del extracto Gentianella nítida. An Fac Med. 2016; 77(4): 333-7.

23. Harnly J. Antioxidant methods. J Food Comp Anal. 2017; 64 (Part 2): 145-146.

24. Granato D, Sousa J, Galvao L, Sávio D. Chemical perspective and criticism on selected analytical methods used to estimate the total content of phenolic compounds in food matrices. Trends in Analytical Chemistry. 2016; 80: 266-279.

25. Cavero S, García-Risco MR, Marín FR, et al. Supercritical fluid extraction of antioxidant compounds from oregano: Chemical and functional characterization via LC-MS and in vitro assays. J of Supercritical Fluids. 2006; 38(1): 62-69.

26. Moghrovyan A, Sahakyan N, Babayan A, Chichoyan N, Petrosyan M, Trchounian A. Essential Oil and Ethanol Extract of Oregano (Origanum vulgare L.) from Armenian Flora as a Natural Source of Terpenes, Flavonoids and other Phytochemicals with Antiradical, Antioxidant, Metal Chelating, Tyrosinase Inhibitory and Antibacterial Activity. Curr Pharm Des. 2019; 25(16):1809-1816.

27. Fierascu RC, Ortan A, Fierascu IC, Fierascu I. In vitro and in vivo evaluation of antioxidant properties of wild-growing plants. A short review. Curr. Opin. Food Sci. 2018; 24: 1-8.

28. Teixeira B, Marques A, Ramos C, et al. Chemical composition and bioactivity of different oregano (Origanum vulgare) extracts and essential oil. J Sci Food Agric. 2013; 93(11):2702-14.

29. Méabed EMH, El-Sayed NM, Abou-Sreea AIB, Roby 
MHH. Chemical analysis of aqueous extracts of Origanum majorana and Foeniculum vulgare and their efficacy on Blastocystis spp. cysts. Phytomedicine. 2018; 43:158-163.

30. Chun SS, Vattem DA, Lin YT, Shetty K. Phenolic antioxidants from clonal oregano (Origanum vulgare) with antimicrobial activity against Helicobacter pylori. Process Biochemistry. 2005; 40(2):809-816.
31. Fenoy I. Influencia de los antioxidantes, ascorbato de sodio y alfa tocoferol, en la fuerza de adhesión a esmalte previamente blanqueado con dentífricos blanqueadores. Tesis Doctoral. Barcelona, España: Universitat Internacional de Catalunya; 2017. pp. 155.

Recibido : 11-04-2020

Aceptado : 16-12-2020 